\title{
The predictive role of biomarkers for mortality in COVID-19 patients
}

\author{
Savrun, A. ${ }^{1}{ }^{*}$, Aydin, I.E. ${ }^{1}$, Savrun, S.T. ${ }^{1}$, Karaman, U. ${ }^{2}$ \\ ${ }^{1}$ Department of Emergency Medicine, Ordu University Faculty of Medicine, Ordu, Turkey \\ ${ }^{2}$ Department of Parasitology, Ordu University Faculty of Medicine, Ordu, Turkey \\ *Corresponding author: atakan4601@hotmail.com
}

\section{ARTICLE HISTORY}

Received: 18 June 2021

Revised: 23 July 2021

Accepted: 23 July 2021

Published: 30 August 2021

\begin{abstract}
Many biomarkers are used in addition to radiologic examinations to determine the severity of COVID-19. This study aims to determine WBC, neutrophil, lymphocyte, platelet, D-dimer, CRP, AST, ALT, LDH, PT, APTT, INR, urea, creatinine, lactate, and ferritin levels in COVID-19 patients and the effect of their changes on mortality rate. The study was conducted between 11 March 2020 and 31 August 2020 (during the COVID-19 pandemic). A total of 502 patients older than 18 years who presented with suspected COVID-19 were included in the study. Of these 502 patients who applied to the hospital, 229(45.6\%) were male and $273(54 \%)$ were female. $301(60 \%)$ patients were diagnosed with COVID-19 through computed tomography and PCR tests. 201(40\%) patients with negative test results constituted the control group. Patients with positive test results $48.2 \%(n=145)$ were men, and $51.8 \%(n=156)$ were women. The median age of the patients was $51 \pm 25$ years. The patients tested positive for COVID-19 were divided into three groups as outpatients $(26.9 \%)$, inpatients $(68.8 \%)$, and intensive care unit patients $(4.3 \%)$. The mortality rate of the patients followed via the patient follow-up system after 30 days was determined as $2.7 \%$. The biomarker values of patients examined in this study tested negative and positive for COVID-19 were compared. In the study, D-dimer, ferritin, Lactate, AST, ALT, LDH, Urea, Creatinine, APTT, and INR levels were found to be higher in the positive tested patients than the negative ones. In the study, it was concluded that neutrophil, lymphocyte, CRP, and ferritin ratios should also be followed in the follow-up phase of the disease. It is important that additional measures should be taken in cases when these biomarkers increase by following the values of the patients who started taking treatment. Also, the ratio of biomarkers is crucial in determining whether the treatment has been effective or not.
\end{abstract}

Keywords: COVID-19; biomarkers; ferritin; CRP; NLR.

\section{INTRODUCTION}

The new coronavirus, SARS-CoV-2 (COVID-19), was first detected in China, in Hubei Wuhan, in December 2019. COVID19, the coronavirus 2019 disease, spread throughout the world and was declared a pandemic by the World Health Organization (WHO) on March 11, 2020. COVID-19 has killed many people worldwide as a result of pneumonia and other complications. Patients with COVID-19 experience many symptoms, such as fever, shortness of breath, cough, sputum, nausea, vomiting, diarrhea, myalgia, loss of taste and smell, headache, anorexia, and weakness. In addition, pneumonia can lead to death by causing acute respiratory distress syndrome. Many biomarkers are used in addition to radiologic examinations to determine the severity of the disease. These biomarkers are useful in predicting the prognosis of patients and in managing treatment processes (Montrief et al., 2020).
Some biomarkers are commonly used in COVID-19. These are the levels of white blood cell (WBC), neutrophil, lymphocyte, platelet, D-dimer, c-reactive protein (CRP), procalcitonin, Aspartate Aminotransferase (AST), Alanine Aminotransferase (ALT), lactate dehydrogenase (LDH), urea, creatinine, ferritin, lactate, Prothrombin Time (PT), Activated Partial Thromboplastin Time (APTT) and International Normalized Rate (INR). In the resource materials, different parameters were investigated in different studies, and it was reported that it may affect mortality in the patients with COVID-19. Accordingly, it was aimed to determine WBC, neutrophil, lymphocyte, platelet, D-dimer, CRP, AST, ALT, LDH, PT, APTT, INR, urea, creatinine, lactate, and ferritin levels in the patients with COVID-19 and to determine the effect of their change on mortality rate. Therefore, it will make possible an evaluation with a more holistic approach, and it will be easier to follow the health status of the patients according to the obtained data. 


\section{MATERIALS AND METHODS}

\section{Ethics committee approval}

Ethical approval was obtained from Ordu University Clinical Research Ethics Committee (Date: 10 December 2021, Decision Number: 2020/256).

\section{Patients}

The population of this study consists of patients admitted to Ordu University Department of Emergency Medicine between March 11 and August 31, 2020. The patients admitted with fever and respiratory-related symptoms and considered infected with COVID-19 by imaging methods were evaluated. Computed tomography (CT) and PCR analysis were carried out on 502 patients admitted to the emergency service. In addition, blood samples were routinely taken from each patient for WBC, neutrophil, lymphocyte, platelet, D-dimer, CRP, AST, ALT, LDH, PT, APTT, INR, urea, creatinine, lactate, and ferritin tests. Afterward, the routine treatment protocol of the Ministry of Health was applied to the patients who were positive for COVID-19. The inpatients were followed up for 30 days, and their health status was evaluated.

\section{Data Collection}

The date of admission to the emergency department for each patient enrolled in the study, as well as the age, gender, comorbidities (e.g., hypertension, diabetes mellitus (DM), chronic obstructive pulmonary disease/asthma (COPD), coronary artery disease (CAD), and SARS-CoV-2 PCR analysis obtained in the emergency department, were documented. In addition, WBC, neutrophil, lymphocyte, platelet, D-dimer, CRP, AST, ALT, LDH, PT, APTT, INR, urea, creatinine, lactate, and ferritin, patient discharge details (including outpatient unit follow-up visits), the hospitalization status (inpatient, intensive care unit), and the 30-day mortality status were retrospectively collected using a hospital computerized database.

\section{Laboratory Analysis}

Blood tests obtained from peripheral venous blood samples of the patients were retrospectively analyzed. Complete blood count analysis (e.g., WBC, neutrophil, lymphocyte) of the patients was studied using the Sysmex XN-1000 device. Biochemical parameters (e.g., the CRP) of the patients were studied with the Roche Cobas c 701 device. Ferritin tests of the patients were conducted with the Roche Cobas e 801 device. Combined throat-nose swabs were taken from the patients in the emergency service, and SARS-CoV-2 PCR analyses were performed in the Ordu Clinical Microbiology Laboratory.

\section{Ethical Considerations}

Before the study, ethical approval was obtained from the Ordu University Local Clinical Research Ethics Committee (Date: 10 December 2020; Decision number 2020/256). Because the study was conducted retrospectively, the need for informed consent forms from patients was waived. The study was conducted in accordance with the Declaration of Helsinki.

\section{Statistical Analysis}

The data analysis was carried out by using SPSS v26 software (IBM Inc., Chicago, IL, USA). The data was given in numbers and percentages. The relationship between the types of the comorbidities and treatment settings or the patient mortality was investigated by using the Chi-square t-test. The relationship between biomarkers and mortality was examined by using the Mann-Whitney $U$ test. Before the analysis, the data were tested for normality by using the Kolmogorov-Smirnov test and for variance homogeneity by using the Levene test. The statistical evaluation was considered significant when $\mathrm{P}<0.05$.

\section{RESULTS}

Of 502 patients admitted to the emergency service, 229 (45.6\%) were male, and 273 (54\%) were female. Of these, 301 (60\%) were found to be tested positive for COVID-19 through computed tomography (CT) and PCR analyses. 201 (40\%) patients who were tested negative for COVID-19 constituted the control group.

The patients tested positive for COVID-19 48.2\% ( $n=145)$ were men, and $51.8 \%(n=156)$ were women. The median age of the patients was $51 \pm 25$ years. The patients tested positive for COVID-19 were divided into three groups as outpatients $(26.9 \%)$, inpatients $(68.8 \%)$, and intensive care unit patients (4.3\%). The mortality rate of the patients followed via the patient follow-up system after 30 days was determined as $2.7 \%$.

We did not find a statistically significant relationship between the gender of the patients and the follow-up status. In addition, we did not find a significant relationship between gender and 30-day mortality. We found a statistically significant relationship between the presence of DM $(p<0.05), H T(p<0.001)$, COPD $(p<0.001)$, or CAD $(p<0.001)$ and 30-day mortality. In addition, the presence of HT $(p<0.01)$, COPD $(p<0.01)$, or CAD $(p<0.05)$ was significantly related to the follow-up status (Table 1). The biomarker values of patients examined in this study tested negative and positive for COVID-19 are given in Table 2. According to the Mann Whitney-U test, there is a significant difference between the patients tested positive and the patients tested negative in the ratios of D-dimer, CRP, ferritin, Lactate, AST, ALT, LDH, Urea, Creatinine, APTT and INR $(P<0.05)$ (Table 2). According to the table, D-dimer, ferritin, Lactate, AST, ALT, LDH, Urea, Creatinine, APTT, and INR were found to be higher in the positive tested patients than the negative ones. Additionally, there is a statistically significant relationship between age $(p<0.001)$, neutrophil $(p<0.05)$, Iymphocyte $(p<0.01)$, CRP $(p<0.01)$, ferritin $(p<0.05)$ values, and 30 -day mortality. There is a statistically significant relationship between age

Table 1. Demographic data of patients

\begin{tabular}{llcc}
\hline & & $\mathrm{n}$ & $\%$ \\
\hline GENDER & Female & 273 & 54,4 \\
\multirow{2}{*}{ MORTALITY } & Male & 229 & 45,6 \\
& Positive & 10 & 2,0 \\
TREATMENT & Negative & 492 & 98,0 \\
& Outdoor & 266 & 53,0 \\
& Inpatient & 222 & 44,2 \\
DM & Intensive care & 14 & 2,8 \\
& Positive & 46 & 9,2 \\
HT & Negative & 456 & 90,8 \\
& Positive & 65 & 12,9 \\
CAD & Negative & 437 & 87,1 \\
& Positive & 32 & 6,4 \\
COPD & Negative & 470 & 93,6 \\
& Positive & 26 & 5,2 \\
& Negative & 476 & 94,8 \\
\hline
\end{tabular}


Table 2. Biomarkers values

\begin{tabular}{|c|c|c|c|c|c|}
\hline & \multicolumn{2}{|c|}{ SARS-CoV-2 NEGATIVE } & \multicolumn{2}{|c|}{ SARS-CoV-2 POSITIVE } & \multirow{2}{*}{$P$} \\
\hline & MEDIAN & IQR & MEDIAN & IQR & \\
\hline WBC & 6,29 & 3,73 & 6,27 & 3,70 & ,574 \\
\hline NEUTROPHIL & 2,11 & 1,90 & 2,76 & 2,76 & ,001 \\
\hline LENPHOSITE & 1,59 & 1,15 & 1,48 & 1,00 & ,021 \\
\hline PLT & 224 & 87 & 217 & 87 & , 107 \\
\hline D-DIMER & 0,16 & 0,27 & 0,26 & 0,31 & ,000 \\
\hline CRP & 0,46 & 0,94 & 1,36 & 4,92 & ,000 \\
\hline FERRITIN & 63,1 & 105,4 & 130 & 247 & ,000 \\
\hline LAC & 0,90 & 0,6 & 1,3 & 1 & ,000 \\
\hline AST & 19 & 11 & 21 & 11 &, 002 \\
\hline ALT & 15 & 13 & 19 & 16 & ,000 \\
\hline LDH & 196 & 338 & 206 & 77 & ,025 \\
\hline BUN & 9,2 & 2,7 & 11,7 & 10,95 & ,000 \\
\hline CRE & 0,67 & 0,18 & 0,86 & 0,29 & ,000 \\
\hline PT & 14,2 & 3,7 & 13,9 & 3,5 & ,654 \\
\hline PTT & 29,2 & 6,2 & 29,9 & 6,75 & ,002 \\
\hline INR & 0,95 & 0,77 & 1,6 & 0,65 & ,000 \\
\hline
\end{tabular}

Mann Whitney $-U, p<0,005$ is important.

$(p<0.001), W B C(p<0.001)$, neutrophil $(p<0.01), \operatorname{CRP}(p<0.001)$ values and outpatient/inpatient unit follow-up. There is a statistically significant relationship between patients' age $(p<0.05)$, CRP $(p<0.05)$, ferritin $(p<0.01)$ values and inpatient unit/ICUP follow-up. A statistically significant relationship was found between the patients' age and all biomarkers except ferritin and outpatient unit/ICUP follow-up (Table 3).

\section{DISCUSSION}

The main mechanism of COVID-19 infection, the binding of the virus to membrane-bound form of angiotensin-converting enzyme 2 (ACE2) and the internalization of the complex by the host cell. The coronavirus spike (S) protein binds strongly with ACE2 and the serine protease TMPRSS2 (Tai et al., 2020) ACE2 and TMPRSS2 are not only found in the lungs but also in many parts of the body. They are also found in the small intestine epithelium, esophagus, liver, colon, blood vessels, heart, kidneys, ovaries, and testicles (D'Amico et al., 2020). Therefore, systemic effects can be seen in COVID-19 infection.

There is a need for biomarkers that can help risk stratification of patients, predict patients' prognosis early, and show response to treatment and mortality. There are biomarkers used in risk classification and disease prognosis of other diseases (Henry et al., 2020). COVID-19 can also cause systemic effects with the above-mentioned mechanisms. For this reason, they reported in the literature that some biomarkers can be used for this purpose. These include WBC, neutrophil, lymphocyte, neutrophil-to-lymphocyte ratio (NLR), C-reactive protein (CRP), ferritin (Du et al., 2020; Zhang G et al., 2020). In patients diagnosed with COVID-19 infection, depending on the viral effect and functional exhaustion; It has been reported that there is a decrease in the number of CD8 + T cells, cytotoxic lymphocytes (CTLs) and the total number of B cells (Cossarizza et al., 2020). In another study, they reported that patients with more severe COVID-19 had low lymphocyte, higher leukocyte count and higher NLR values (Chen et al., 2020). According to another study, COVID19 patients with severe and fatal symptoms have been reported to have increased WBC, decreased lymphocyte, and platelet counts (Henry et al., 2020). In the study of Xu et al.

Table 3. The effect of age and biomarkers on patient follow-up and mortality

\begin{tabular}{|c|c|c|c|c|c|c|c|c|c|c|}
\hline & & \multirow{2}{*}{$\mathrm{n}$} & \multirow{2}{*}{$\%$} & \multicolumn{4}{|c|}{ TREATMENT } & \multicolumn{3}{|c|}{ MORTALITY } \\
\hline & & & & Outdoor & Inpatient & Intensive care & $p$ & Negative & positive & $\mathrm{p}$ \\
\hline \multirow[t]{4}{*}{ GENDER } & Male & 229 & $45,6 \%$ & 111 & 110 & 8 & 153 & 224 & 5 & ,779 \\
\hline & & & & $48,5 \%$ & $48,0 \%$ & $3,5 \%$ & & $97,8 \%$ & $2,2 \%$ & \\
\hline & Female & 273 & $54,4 \%$ & 155 & 112 & 6 & & 268 & 5 & \\
\hline & & & & $56,8 \%$ & $41,0 \%$ & $2,2 \%$ & & $98,2 \%$ & $1,8 \%$ & \\
\hline \multirow[t]{4}{*}{ DM } & Negative & 456 & $90,8 \%$ & 247 & 198 & 11 & 102 & 449 & 7 & 021 \\
\hline & & & & $54,2 \%$ & $43,4 \%$ & $2,4 \%$ & & $98,5 \%$ & $1,5 \%$ & \\
\hline & Positive & 46 & $9,2 \%$ & 19 & 24 & 3 & & 43 & 3 & \\
\hline & & & & $41,3 \%$ & $52,2 \%$ & $6,5 \%$ & & $93,5 \%$ & $6,5 \%$ & \\
\hline \multirow[t]{4}{*}{$\mathrm{HT}$} & Negative & 437 & $87,1 \%$ & 246 & 184 & 7 & ,000 & 434 & 3 & , 000 \\
\hline & & & & $56,3 \%$ & $42,1 \%$ & $1,6 \%$ & & $99,3 \%$ & $0,7 \%$ & \\
\hline & Positive & 65 & $12,9 \%$ & 20 & 38 & 7 & & 58 & 7 & \\
\hline & & & & $30,8 \%$ & $58,5 \%$ & $10,8 \%$ & & $89,2 \%$ & $10,8 \%$ & \\
\hline \multirow[t]{4}{*}{$C A D$} & Negative & 470 & $93,6 \%$ & 260 & 200 & 10 & ,000 & 465 & 5 & ,000 \\
\hline & & & & $55,3 \%$ & $42,6 \%$ & $2,1 \%$ & & $98,9 \%$ & $1,1 \%$ & \\
\hline & Positive & 32 & $6,4 \%$ & 6 & 22 & 4 & & 27 & 5 & \\
\hline & & & & $18,8 \%$ & $68,8 \%$ & $12,5 \%$ & & $84,4 \%$ & $15,6 \%$ & \\
\hline \multirow[t]{4}{*}{ COPD } & Negative & 476 & $94,8 \%$ & 262 & 200 & 14 & ,000 & 471 & 5 & ,000 \\
\hline & & & & $55,0 \%$ & $42,0 \%$ & $2,9 \%$ & & $98,9 \%$ & $1,1 \%$ & \\
\hline & Positive & 26 & $5,2 \%$ & 4 & 22 & 0 & & 21 & 5 & \\
\hline & & & & $15,4 \%$ & $84,6 \%$ & $0,0 \%$ & & $80,8 \%$ & $19,2 \%$ & \\
\hline
\end{tabular}

Kruskal-Wallis test $*<0.05 * *<0.01 * * *<0.001$ WBC; White blood cell, NLR; neutrophil lymphocyte ratio, CRP; C-reactive protein, ICU; Intensive care unit. 
(2020), it was reported that patients with a more serious clinical course or who died had a lower T lymphocyte count (Bo et al., 2020). In another study, it was reported that as the NLR increased, the duration of hospitalization and mortality rates increased (Liu et al., 2020). In this study, unlike other studies, WBC, neutrophil, lymphocyte, platelet, D-dimer, CRP, AST, ALT, LDH, PT, APTT, INR, urea, creatinine, lactate, and ferritin levels were examined in COVID-19 patients and higher rates were found compared to the ones tested negative. In addition, according to the Table 3 , it was observed that there is a significant increase in age, neutrophil, lymphocyte, CRP, and ferritin ratios and a significant increase in the death rate at 30-day follow-up. It shows that neutrophil, lymphocyte, CRP, and ferritin ratios should be monitored in COVID-19 patients and necessary precautions should be taken in cases when there is an increase. In addition, similar to other studies, it was determined that mortality increases with age.

CRP is a plasma protein which produced by the liver. Various inflammatory conditions have been reported that there is a relationship between the severity of the disease and CRP levels (Gong et al., 2020). Zhang et al. reported in their study that the CRP value increased in COVID-19 patients. In addition, while the median value of CRP in COVID-19 surviving patients was $40 \mathrm{mg} / \mathrm{L}$. This value was found as 125 $\mathrm{mg} / \mathrm{L}$ in those who died. In the same study, it was reported that CRP was strongly correlated with the severity of COVID19 and prognosis (Zhang et al., 2020). In addition, it was reported that with the increase of CRP in COVID-19, ARDS development and death may be seen (Terpos et al., 2020). In our study, it was determined that hospitalization and mortality increased as the CRP value increased. Therefore, this situation shows that CRP rates should be followed in COVID-19 patients.

Ferritin is an iron-storing protein, and its serum level reflects the normal iron level and helps in the diagnosis of iron deficiency anemia. Also, the level of circulating ferritin increases during viral infections and may be an indicator of viral replication (Li et al., 2020). Ferritin is a biomarker that contributes to cytokine storm by promoting the expression of multiple proinflammatory mediators (Ponti et al., 2020). It was reported that ferritin level increased in tables where cytokine storm was seen, such as COVID-19 (Huang et al., 2020). It was reported in a study that the increase in ferritin level was associated with clinical worsening of COVID-19 and even with ARDS and mortality (Wu et al., 2020). Another study reported that serum ferritin concentration increased in patients with high mortality risk (Dimopoulos et al., 2020). In our study, it was found that ferritin plays a significant role in determining the admission to intensive care unit in patients with COVID-19. In addition, a significant relationship was found between ferritin and mortality in patients with COVID-19.

As a result in the study, it was concluded that the neutrophil, lymphocyte, CRP, and ferritin ratios tested to support the diagnosis should be followed in the later phases of the disease. Additional measures should be taken in cases where these biomarkers increase by controlling the values of the patients who started taking treatment. In addition, these parameters maintain that data will be obtained about whether the treatment is effective or not thus facilitating the follow-up of the disease. Also, as age increases, the mortality rate increases, so preventive health services should be activated in these groups.

Lack of follow-up data, single blood sampling and small sample size are the limitations of our study since it is a retrospective study. To clarify the underlying mechanisms, it is necessary to confirm these findings in future studies with larger sample sizes.

\section{Data availability}

The data used in this study will be provided on demand.

\section{ACKNOWLEDGMENTS}

The authors would like to express their appreciation for all the health workers to combat the SARS-CoV-2 outbreak. This study did not receive financial support.

\section{Conflict of interest statement}

The author declares that they have no conflict of interests.

\section{REFERENCES}

Bo, X., Fan, C.Y., Wang, A.L., Zou, Y.L., Yu, Y.H., Cong, H.E., Wenguang, X.I.A., Ji Xian, Z.H.A.N.G. \& Qing, M.I.A.O. (2020). Suppressed $T$ cell-mediated immunity in patients with COVID-19: a clinical retrospective study in Wuhan, China. Journal of Infection 81: e51-e60. https://doi.org/10.1016/ j.jinf.2020.04.012

Chen, N., Zhou, M., Dong, X., Qu, J., Gong, F., Han, Y., Qiu, Y., Wang, J., Liu, Y. \& Wei, Y. (2020). Epidemiological and clinical characteristics of 99 cases of 2019 novel coronavirus pneumonia in Wuhan, China: a descriptive study. The Lancet 395: 507-513. https://doi.org/10.1016/ S0140-6736(20)30566-3

Cossarizza, A., De Biasi, S., Guaraldi, G., Girardis, M., Mussini, C. \& Group, M.C.W. (2020). SARS CoV 2, the virus that causes COVID 19: Cytometry and the new challenge for global health. Cytometry 97: 340. https://doi.org/10.1002/cyto. a. 24002

D’Amico, F., Baumgart, D.C., Danese, S. \& Peyrin-Biroulet, L. (2020). Diarrhea during COVID-19 infection: pathogenesis, epidemiology, prevention and management. Clinical Gastroenterology and Hepatology 18: 1663-1672. https:// doi.org/10.1016/j.cgh.2020.04.001

Dimopoulos, G., de Mast, Q., Markou, N., Theodorakopoulou, M., Komnos, A., Mouktaroudi, M., Netea, M.G., Spyridopoulos, T., Verheggen, R.J. \& Hoogerwerf, J. (2020). Favorable anakinra responses in severe COVID19 patients with secondary hemophagocytic lymphohistiocytosis. Cell Host \& Microbe 28: 117-123. https:// doi.org/10.1016/j.chom.2020.05.007

Du, R.H., Liang, L.R., Yang, C.Q., Wang, W., Cao, T.Z., Li, M., Guo, G.Y., Du, J., Zheng, C.L. \& Zhu, Q. (2020). Predictors of mortality for patients with COVID-19 pneumonia caused by SARS-CoV-2. European Respiratory Journal 55: 2000524 https://doi.org/10.1183/13993003.00524-2020

Gong, J., Dong, H., Xia, S.Q., Huang, Y.Z., Wang, D., Zhao, Y., Liu, W., Tu, S., Zhang, M. \& Wang, Q. (2020). Correlation analysis between disease severity and inflammation-related parameters in patients with COVID-19 pneumonia. MedRxiv [Preprint] https://doi.org/10.1101/2020.02.25. 20025643

Henry, B.M., De Oliveira, M.H.S., Benoit, S., Plebani, M. \& Lippi, G. (2020). Hematologic, biochemical and immune biomarker abnormalities associated with severe illness and mortality in coronavirus disease 2019 (COVID-19): a meta-analysis. Clinical Chemistry and Laboratory Medicine (CCLM) 58: 1021-1028. https://doi.org/10.1515/cclm-20200369

Huang, C., Wang, Y., Li, X., Ren, L., Zhao, J., Hu, Y., Zhang, L., Fan, G., Xu, J. \& Gu, X. (2020). Clinical features of patients infected with 2019 novel coronavirus in Wuhan, China. The Lancet 395: 497-506. https://doi.org/10.1016/S01406736(20)30183-5 
Li, Y., Hu, Y., Yu, J. \& Ma, T. (2020). Retrospective analysis of laboratory testing in 54 patients with severe-or criticaltype 2019 novel coronavirus pneumonia. Laboratory Investigation 100: 794-800. https://doi.org/10.1038/s41374020-0431-6

Liu, Y., Du, X., Chen, J., Jin, Y., Peng, L., Wang, H.H., Luo, M., Chen, L. \& Zhao, Y. (2020). Neutrophil-to-lymphocyte ratio as an independent risk factor for mortality in hospitalized patients with COVID-19. Journal of Infection 81: e6-e12. https://doi.org/10.1016/j.jinf.2020.04.002

Montrief, T., Ramzy, M., Long, B., Gottlieb, M. \& Hercz, D. (2020). COVID-19 respiratory support in the emergency department setting. The American Journal of Emergency Medicine 38: 2160-2168. https://doi.org/10.1016/j.ajem. 2020.08.001

Ponti, G., Maccaferri, M., Ruini, C., Tomasi, A. \& Ozben, T. (2020). Biomarkers associated with COVID-19 disease progression. Critical Reviews in Clinical Laboratory Sciences 57: 389-399. https://doi.org/10.1080/10408363.2020.1770685

Tai, W., He, L., Zhang, X., Pu, J., Voronin, D., Jiang, S., Zhau, Y. \& Du, L. (2020). Characterization of the receptor-binding domain (RBD) of 2019 novel coronavirus: implication for development of RBD protein as a viral attachment inhibitor and vaccine. Cellular \& Molecular Immunology 17: 613-620. https://doi.org/10.1038/s41423-020-0400-4
Terpos, E., Ntanasis Stathopoulos, I., Elalamy, I., Kastritis, E., Sergentanis, T.N., Politou, M., Psaltopoulou, T., Gerotziafas, G. \& Dimopoulos, M.A. (2020). Hematological findings and complications of COVID 19. American Journal of Hematology 95: 834-847. https://doi.org/10.1002/ajh.25829

Wu, C., Chen, X., Cai, Y., Zhou, X., Xu, S., Huang, H., Zhang, L., Zhou, X., Du, C. \& Zhang, Y. (2020). Risk factors associated with acute respiratory distress syndrome and death in patients with coronavirus disease 2019 pneumonia in Wuhan, China. JAMA Internal Medicine 180: 934-943. https://doi.org/10.1001/jamainternmed.2020.0994

Xu, B., Fan, C.Y., Wang, A.L., Zou, Y.L., Yu, Y.H., He, C., Xia, W.G., Zhang, J.X. \& Miao, Q. (2020). Suppressed T cell-mediated immunity in patients with COVID-19: A clinical retrospective study in Wuhan, China. Journal of Infection 81: e51-e60. https://doi.org/10.1016/j.jinf.2020.04.012

Zhang, G., Zhang, J., Wang, B., Zhu, X., Wang, Q. \& Qiu, S. (2020). Analysis of clinical characteristics and laboratory findings of 95 cases of 2019 novel coronavirus pneumonia in Wuhan, China: a retrospective analysis. Respiratory Research 21: 1-10. https://doi.org/10.1186/s12931-02001338-8

Zhang, J., Dong, X., Cao, Y., Yuan, Y., Yang, Y., Yan, Y., Akdis, C.A. \& Gao, Y.D. (2020). Clinical characteristics of 140 patients infected with SARS CoV 2 in Wuhan, China. Allergy 75: 17301741. https://doi.org/10.1111/all.14238 